\title{
Cross-sectional area and intensity variations of sausage modes ${ }^{\star}$
}

\author{
M. G. Moreels, M. Goossens, and T. Van Doorsselaere ${ }^{\star \star}$
}

\author{
Centre for mathematical Plasma Astrophysics, Mathematics Department, KU Leuven, Celestijnenlaan 200B bus 2400, \\ 3001 Leuven, Belgium \\ e-mail: michael.moreels@wis. kuleuven . be
}

Received 22 March 2013 / Accepted 2 May 2013

\begin{abstract}
Context. The observations obtained using the Rapid Oscillations in the Solar Atmosphere instrument (ROSA) show variations in both cross-sectional area and intensity for magnetic pores in the photosphere.

Aims. We study the phase behaviour between cross-sectional area and intensity variations for sausage modes in a photospheric context. We aim to determine the wave mode by looking at the phase difference between the cross-sectional area and intensity variations. Methods. We used a straight cylinder as a model for the flux tube. The plasma is uniform both inside and outside the flux tube with a possible jump in the equilibrium values at the boundary, the magnetic field is directed along the flux tube. We derived analytic expressions for the cross-sectional area variation and the total intensity variation. Using these analytic expressions, we calculated the phase differences between the cross-sectional area and the intensity variations. These phase differences were then used to identify the wave mode.

Results. We found that for slow sausage modes the cross-sectional area and intensity variations are always in phase, while for fast sausage modes the variations are in antiphase.
\end{abstract}

Key words. magnetohydrodynamics (MHD) - methods: analytical - Sun: photosphere - Sun: oscillations

\section{Introduction}

Solar oscillations were discovered in 1960 (Leighton 1960), and since then there have been numerous observations of wave motions in the solar atmosphere (Stein \& Leibacher 1974). Recently, modern ground- and space-based observational instruments show ubiquitous magnetohydrodynamic (MHD) waves in different regions of the solar atmosphere. In the corona MHD waves were detected using the Extreme ultraviolet Imaging Telescope (EIT) onboard the Solar and Heliospheric Observatory (SOHO; Thompson et al. 1999), and also with the imaging telescope onboard the Transition Region and Coronal Explorer (TRACE) spacecraft (Nakariakov et al. 1999). More recent observations of MHD waves in the corona have been made with the Coronal Multi-Channel Polarimeter (CoMP; Tomczyk et al. 2007), with the Extreme-ultraviolet Imaging Spectrometer (EIS) onboard Hinode (Van Doorsselaere et al. 2008; Banerjee et al. 2009), and with the Solar Dynamics Observatory Atmospheric Imaging Assembly (SDO/AIA; Liu et al. 2010; Morton et al. 2012). Magnetohydrodynamic waves have recently been detected in the photosphere using the Solar Optical Telescope (SOT; Fujimura \& Tsuneta 2009) and using the Rapid Oscillations in the Solar Atmosphere imaging system (ROSA; Morton et al. 2011; Jess et al. 2012a,b).

The nature of MHD waves in sunspots and pores has been thoroughly investigated. Sunspots have been modelled as thin, gravitationally stratified flux tubes (Roberts \& Webb 1978), while the theory of Edwin \& Roberts (1983) described MHD waves in straight, uniform flux tubes and has

\footnotetext{
* Appendix A is available in electronic form at

http: //www . aanda. org

$\star \star$ Postdoctoral fellow of the FWO-Vlaanderen.
}

been extensively used in the solar corona. Dispersion diagrams for the photosphere (Edwin \& Roberts 1983; Moreels \& Van Doorsselaere 2013) show the nature of waves in a photosperic waveguide. In this article we are interested in axisymmetric $m=0$ modes, which should exhibit periodic variations in the cross-sectional area of the flux tube. These periodic variations were first reported by Dorotovič et al. (2008) using observations obtained with the Swedish Solar Telescope (SST). However, there was no information on the variations in intensity. The first simultaneous observations of variations in the crosssectional area and in the intensity were reported by Morton et al. (2011) using data obtained with ROSA. In that article the authors did not indentify the wave mode, i.e. whether it was fast or slow. Here we propose a theoretical framework for identifying the wave mode by looking at the phase difference between the cross-sectional area and the intensity variation.

We study the phase relations between the intensity and the cross-sectional area variation for sausage modes in the photosphere. To keep the mathematical formulation tractable we used a simple model for a flux tube, i.e. uniform plasma both inside and outside the flux tube with a jump in the equilibrium values at the flux-tube boundary (Edwin \& Roberts 1983; Moreels \& Van Doorsselaere 2013). In this simple setup we analytically calculated the cross-sectional area and the integrated intensity variations for different sausage modes, from which we then deduced the phase differences. We also numerically visualised different sausage modes to verify the phase behaviour.

\section{Mathematical formulation}

We model a flux tube as a straight cylinder with radius $R$. The plasma is uniform both inside and outside the cylinder with a possible jump in the equilibrium values at the 
boundary (cf. Edwin \& Roberts 1983). The magnetic field is directed along the axis of the flux tube and is given by $B_{0, i}$ inside the flux tube and $B_{0, \mathrm{e}}$ outside the flux tube. The plasma pressure and density are $p_{0, \mathrm{i}}$ and $\rho_{0, \mathrm{i}}$ inside the flux tube and $p_{0, \mathrm{e}}$ and $\rho_{0, \mathrm{e}}$ outside the flux tube. We assume that the plasma has no background flow, i.e. the equilibrium velocity is $v_{0}=0$ both inside and outside the flux tube. The ideal MHD equations for this equilibrium configuration have been solved in the past (Edwin \& Roberts 1983; Sakurai et al. 1991). The wave modes in such a flux tube can be solved in terms of the radial component of the Lagrangian displacement $\xi_{r}$ and the total Eulerian pressure perturbation $P^{\prime}$. Thus all we need is a link between the crosssectional area, the integrated intensity variations on the one hand and $\xi_{r}, P^{\prime}$ on the other. We point out that this model omits important physics, e.g. density stratification and loop expansion (Osterbrock 1961; Rosenthal et al. 2002; Erdélyi et al. 2007). For this work we are mainly interested in the radial eigenfunctions that need to be integrated over a surface. Previous work (e.g. Andries \& Cally 2011) has shown that the perpendicular eigenfunctions remain similar for slowly expanding flux tubes. Therefore we believe that in a first approximation the use of a uniform flux-tube model is justified.

\subsection{General case}

We begin the derivation in a more general case than the uniform flux tube, namely a flux tube where the equilibrium parameters also vary in the radial direction. To calculate the cross-sectional area and the integrated intensity variations we need to calculate a surface integral over a moving surface. For the cross-sectional area we need to evaluate $\int_{S} \mathrm{~d} S$ and for the integrated intensity we need to evauate $\int_{\mathrm{S}} I \mathrm{~d} S$. We are aware that for the intensity it makes more sense to integrate over the surface where the optical depth $\tau=1$. In Appendix A we show that in a linear theory integrating over a constant $\tau$ surface is the same as integrating over a surface that follows the motion of the plasma. More generally, we show that the difference is second order (in the perturbations) between integrating over a circular cross-section, a co-moving surface, or the $\tau=1$ layer. This also confirms that it is justified to neglect gravity since this would not affect the radial displacement of the wave mode. Figure 1 represents a sketch of the surface of constant optical depth for a slow surface sausage mode. The surface that follows the motion of the plasma has the same shape as this surface, as is shown in Appendix A. In a linear approximation we can model the equation of the surface as $f(r, \varphi, z, t) \equiv z-\xi_{z}(r, \varphi, z=0, t)$. Because we are studying sausage modes, there is no $\varphi$ dependence, and we drop this variable. An elemental surface element $\mathrm{d} S$ is easily related to an elemental surface element in the horizontal plane $\mathrm{d} S_{\mathrm{h}}$, i.e.

$\mathrm{d} S=\mathrm{d} S_{\mathrm{h}} \sqrt{1+\left(\frac{\partial \xi_{z}}{\partial r}\right)^{2}}$

In a linear theory this simplifies to $\mathrm{d} S=\mathrm{d} S_{\mathrm{h}}=r \mathrm{~d} r \mathrm{~d} \varphi$. To calculate the cross-sectional area $S(R)$, we need to evaluate

$S(R)=\int_{\mathrm{S}} \mathrm{d} S$

where $S$ is the surface that follows the motion of the plasma. In the equilibrium state it corresponds to the surface $S_{0}$ that is bounded by the circle $C_{0}$ with radius $R$. In other words, our surface integral becomes a one-dimensional integral where the line elements are moving, i.e.

$S(R)=2 \pi \int_{0}^{R+\xi_{r}(R)} r \mathrm{~d} r$.

A75, page 2 of 9

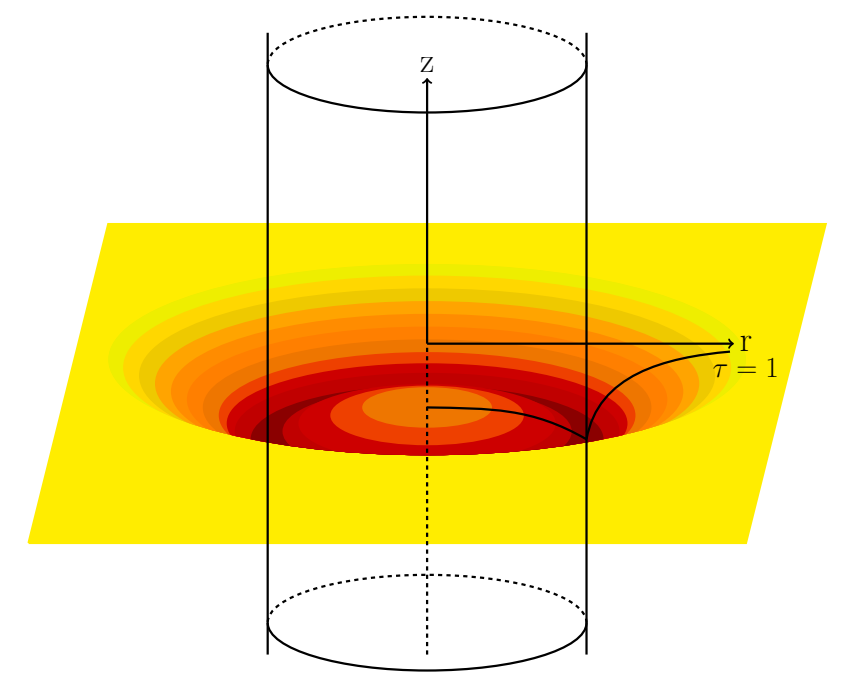

Fig. 1. Sketch of the surface of constant optical depth for a slow surface mode.

If we substitute $r=r_{0}+\xi_{r}\left(r_{0}\right)$, we find

$S(R)=2 \pi \int_{0}^{R}\left(r_{0}+\xi_{r}\left(r_{0}\right)\right)\left(1+\frac{\partial \xi_{r}\left(r_{0}\right)}{\partial r_{0}}\right) \mathrm{d} r_{0}$.

Neglecting higher-order terms shows

$S(R)=2 \pi \int_{0}^{R}\left\{r_{0}+\frac{\partial\left(r_{0} \xi_{r}\right)}{\partial r_{0}}\right\} \mathrm{d} r_{0}$,

resulting in $S(R)=\pi R^{2}+2 \pi R \xi_{r}(R)$. The Lagrangian variation of the cross-sectional area is $\delta S=S(R)-S_{0}=2 \pi R \xi_{r}(R)$. Finally, we normalise the cross-sectional area variation with the equilibrium value to find

$\frac{\delta S}{S_{0}}=2 \frac{\xi_{r}(R)}{R}$.

This equation shows that the cross-sectional area variation depends only on the radial component of the Lagrangian displacement, as we would expect for axisymmetric modes.

Next we calculate the integrated intensity, which is given by

$\mathcal{I}(R)=\int_{\mathrm{S}} \mathcal{I} \mathrm{d} S$,

where $S$ is again the surface that follows the motion of the plasma. As before, the integrated intensity simplifies to a onedimensional integral

$\mathcal{I}(R)=2 \pi \int_{0}^{R+\xi_{r}(R)} \mathcal{I} \mathrm{d} r$.

If we denote $F(\boldsymbol{r}, t)=2 \pi r \mathcal{I}(\boldsymbol{r}, t)$, then

$\mathcal{I}(R)=\int_{0}^{R+\xi_{r}(R)} F(\boldsymbol{r}, t) \mathrm{d} r$.

We know that $\boldsymbol{r}=\boldsymbol{r}_{0}+\boldsymbol{\xi}\left(\boldsymbol{r}_{0}, t\right)$, which leads us to the same substitution as before, namely $r=r_{0}+\xi_{r}\left(r_{0}\right)$ to find

$\mathcal{I}(R)=\int_{0}^{R} F\left(\boldsymbol{r}_{0}+\boldsymbol{\xi}\left(\boldsymbol{r}_{0}, t\right), t\right)\left(1+\frac{\partial \xi_{r}\left(r_{0}\right)}{\partial r_{0}}\right) \mathrm{d} r_{0}$.

We now expand $F\left(\boldsymbol{r}_{0}+\boldsymbol{\xi}\left(\boldsymbol{r}_{0}, t\right), t\right)$ around $\boldsymbol{r}_{0}$, i.e. $F\left(\boldsymbol{r}_{0}+\right.$ $\left.\boldsymbol{\xi}\left(\boldsymbol{r}_{0}, t\right), t\right)=F\left(\boldsymbol{r}_{0}, t\right)+\boldsymbol{\xi}\left(\boldsymbol{r}_{0}, t\right) \cdot \nabla F\left(\boldsymbol{r}_{0}, t\right)$. We can also expand 
$F\left(\boldsymbol{r}_{0}, t\right)$ around the equilibrium to find $F\left(\boldsymbol{r}_{0}, t\right)=F_{0}\left(r_{0}\right)+$ $F^{\prime}\left(\boldsymbol{r}_{0}, t\right)$, where 0 indicates the equilibrium and the prime indicates the Eulerian perturbation. Combining this and neglecting higher-order terms shows

$\mathcal{I}(R)=\int_{0}^{R}\left\{F_{0}\left(r_{0}\right)+F^{\prime}\left(r_{0}\right)+\xi_{r}\left(r_{0}\right) \frac{\partial F_{0}\left(r_{0}\right)}{\partial r_{0}}+F_{0}\left(r_{0}\right) \frac{\partial \xi_{r}\left(r_{0}\right)}{\partial r_{0}}\right\} \mathrm{d} r_{0}$.

This can be integrated to find

$\mathcal{I}(R)=\int_{0}^{R} F_{0}\left(r_{0}\right) \mathrm{d} r_{0}+\int_{0}^{R} F^{\prime}\left(r_{0}\right) \mathrm{d} r_{0}+\left[F_{0}\left(r_{0}\right) \xi_{r}\left(r_{0}\right)\right]_{0}^{R}$.

The Lagrangian integrated intensity variation is $\delta \mathcal{I}=\mathcal{I}-\mathcal{I}_{0}$, i.e.

$\delta \mathcal{I}=\int_{0}^{R} F^{\prime}\left(r_{0}\right) \mathrm{d} r_{0}+\left[F_{0}\left(r_{0}\right) \xi_{r}\left(r_{0}\right)\right]_{0}^{R}$.

When we substitute $F\left(r_{0}\right)$, we find

$\delta \mathcal{I}=2 \pi \int_{0}^{R} \mathcal{I}^{\prime}\left(r_{0}\right) r_{0} \mathrm{~d} r_{0}+2 \pi R \mathcal{I}_{0}(R) \xi_{r}(R)$.

Normalising with $\mathcal{I}_{0}$ shows

$\frac{\delta \mathcal{I}}{\mathcal{I}_{0}}=\frac{\int_{0}^{R} \mathcal{I}^{\prime}\left(r_{0}\right) r_{0} \mathrm{~d} r_{0}}{\int_{0}^{R} \mathcal{I}_{0}\left(r_{0}\right) r_{0} \mathrm{~d} r_{0}}+\frac{R \mathcal{I}_{0}(R) \xi_{r}(R)}{\int_{0}^{R} \mathcal{I}_{0}\left(r_{0}\right) r_{0} \mathrm{~d} r_{0}}$.

This equation shows that the integrated intensity variation depends on $\mathcal{I}_{0}, \mathcal{I}^{\prime}$, and $\xi_{r}$. In the uniform case the integrals including $\mathcal{I}_{0}$ will be easy to calculate, therefore we only need a link between the Eulerian perturbation of the intensity $I^{\prime}$ and the eigenfunctions $\xi_{r}, P^{\prime}$.

We use the same method to calculate the magnetic flux $\phi_{B}$, which is equal to

$\phi_{B}(R)=\int_{\mathrm{S}} \boldsymbol{B} \cdot \mathbf{1}_{n} \mathrm{~d} S$,

where $S$ is again the surface that follows the motion of the plasma. We easily find that

$\mathbf{1}_{n}=\frac{1}{\sqrt{1+\left(\frac{\partial \xi_{z}}{\partial r}\right)^{2}}}\left(-\frac{\partial \xi_{z}}{\partial r} \mathbf{1}_{r}+\mathbf{1}_{z}\right)$.

Because the equilibrium magnetic field $\boldsymbol{B}_{0}$ has no radial component $\boldsymbol{B} \cdot \mathbf{1}_{n}=B_{z}$. As before, $\mathrm{d} S=r \mathrm{~d} r \mathrm{~d} \varphi$, thus

$\phi_{B}(R)=2 \pi \int_{0}^{R+\xi_{r}(R)} B_{z} r \mathrm{~d} r$.

When we denote $F(\boldsymbol{r}, t)=2 \pi r B_{z}(\boldsymbol{r}, t)$ we can use the same reasoning as for the intensity to find

$\delta \phi_{B}=2 \pi \int_{0}^{R} B_{z}^{\prime}\left(r_{0}\right) r_{0} \mathrm{~d} r_{0}+2 \pi R B_{z, 0}(R) \xi_{r}(R)$,

which after normalising becomes

$\frac{\delta \phi_{B}}{\phi_{B, 0}}=\frac{\int_{0}^{R} B_{z}^{\prime}\left(r_{0}\right) r_{0} \mathrm{~d} r_{0}}{\int_{0}^{R} B_{z, 0}\left(r_{0}\right) r_{0} \mathrm{~d} r_{0}}+\frac{R B_{z, 0}(R) \xi_{r}(R)}{\int_{0}^{R} B_{z, 0}\left(r_{0}\right) r_{0} \mathrm{~d} r_{0}}$.

This equation shows that the magnetic flux variation depends on $B_{z, 0}, B_{z}^{\prime}$, and $\xi_{r}$. In the uniform case the integrals including $B_{z, 0}$ will be easy to calculate, therefore we only need a link between the Eulerian perturbation of the $z$-component of the magnetic field $B_{z}^{\prime}$ and the eigenfunctions $\xi_{r}, P^{\prime}$.

We still need a link between the intensity and the eigenfunctions $\xi_{r}$ and $P^{\prime}$. Using the fact that the photosphere is optically thick and by assuming local thermodynamic equilibrium, we find that the continuum intensity is given by (e.g. Rutten 2003)

$\mathcal{I}=\frac{2 h v^{3}}{c^{2}} \frac{1}{\exp \left(h v / k_{\mathrm{B}} T\right)-1}$,

where $h$ is the Planck constant, $k_{\mathrm{B}}$ is the Boltzmann constant, $T$ is the temperature of the plasma, $v$ is the frequency, and $c$ is the speed of light. We now linearise this expression. We know that $T(r)=T_{0}(r)+T^{\prime}(r, t)$ with $T^{\prime} / T_{0} \ll 1$, where 0 indicates the equilibrium value while the prime denotes the Eulerian perturbed value. Using this, we find

$\mathcal{I}=\mathcal{I}_{0}(r)+\frac{h v}{k_{\mathrm{B}} T_{0}(r)} \mathcal{I}_{0} \frac{T^{\prime}}{T_{0}}$,

where $\mathcal{I}_{0}$ is given by

$\mathcal{I}_{0}(r)=2 \frac{h v^{3}}{c^{2}} \exp \left(-\frac{h v}{k_{\mathrm{B}} T_{0}(r)}\right)$.

We linearise the ideal gas law to find

$\frac{T^{\prime}}{T_{0}}=\frac{p^{\prime}}{p_{0}}-\frac{\rho^{\prime}}{\rho_{0}}$.

Conservation of mass and energy shows

$\frac{\rho^{\prime}}{\rho_{0}}=-\nabla \cdot \boldsymbol{\xi}-\frac{1}{\rho_{0}} \boldsymbol{\xi} \cdot \nabla \rho_{0}$

$\frac{p^{\prime}}{p_{0}}=\gamma \frac{\rho^{\prime}}{\rho_{0}}+\gamma \frac{1}{\rho_{0}} \boldsymbol{\xi} \cdot \nabla \rho_{0}-\frac{1}{p_{0}} \boldsymbol{\xi} \cdot \nabla p_{0}$,

where $\gamma$ is the ratio of specific heats. Combining the above equations results in

$\frac{T^{\prime}}{T_{0}}=-(\gamma-1) \nabla \cdot \boldsymbol{\xi}+\left(\frac{1}{\rho_{0}} \boldsymbol{\xi} \cdot \nabla \rho_{0}-\frac{1}{p_{0}} \boldsymbol{\xi} \cdot \nabla p_{0}\right)$.

Thus Eq. (4) becomes

$\frac{\mathcal{I}^{\prime}}{\mathcal{I}_{0}}=\frac{h v}{k_{\mathrm{B}} T_{0}}\left(-(\gamma-1) \nabla \cdot \boldsymbol{\xi}+X(r) \xi_{r}\right)$,

where $X(r)$ is given by

$X(r)=\frac{1}{\rho_{0}} \frac{\mathrm{d} \rho_{0}}{\mathrm{~d} r}-\frac{1}{p_{0}} \frac{\mathrm{d} p_{0}}{\mathrm{~d} r}$.

We would like to stress that Eq. (5) shows that in a uniform plasma $\mathcal{I}^{\prime}$ is proportional to $\nabla \cdot \boldsymbol{\xi}$, which coincides with the equation in Moreels \& Van Doorsselaere (2013). However, in a non-uniform plasma $\mathcal{I}^{\prime}$ also has a contribution depending on $\xi_{r}$. Using Sakurai et al. (1991), we find an expression for $\nabla \cdot \boldsymbol{\xi}$ in terms of the total pressure perturbation $P^{\prime}$, meaning that we have a link between the intensity and the eigenfunctions $\xi_{r}, P^{\prime}$.

The $z$-component of the Eulerian perturbation of the magnetic field $B_{z}^{\prime}$ can be expressed with the use of $\xi_{r}$ and $P^{\prime}$, i.e.

$B_{z}^{\prime}=\mathrm{i} k B_{z, 0} \xi_{z}-B_{z, 0} \nabla \cdot \boldsymbol{\xi}-\frac{\mathrm{d} B_{z, 0}}{\mathrm{~d} r} \xi_{r}$

where we only need an expression for the z-component of the Lagrangian displacement $\xi_{z}$. We have

$\rho_{0}\left(\omega^{2}-k^{2} c_{\mathrm{A}}^{2}\right) \xi_{z}=\mathrm{i} k P^{\prime}+\mathrm{i} k \frac{B_{0}^{2}}{\mu} \nabla \cdot \xi$.

We can express $\nabla \cdot \xi$ in terms of the eigenfunctions $\xi_{r}, P^{\prime}$ (Sakurai et al. 1991) and combining with the above equations we thus have the $z$-component of the Eulerian perturbation of the magnetic field $B_{z}^{\prime}$ in terms of the eigenfunctions $\xi_{r}, P^{\prime}$. 


\subsection{Uniform tube}

Up to now the derivation has been general in the sense that the equilibrium quantities were allowed to vary in the radial direction. Now we assume a uniform flux tube, thus $X(r)=0$ and in Eq. (2) the integral $\int_{0}^{R} \mathcal{I}_{0}\left(r_{0}\right) r_{0} \mathrm{~d} r_{0}$ can be evaluated to find $\mathcal{I}_{0} R^{2} / 2$. In the uniform case Eq. (2) becomes

$\frac{\delta \mathcal{I}}{\mathcal{I}_{0}}=\frac{-2(\gamma-1)}{R^{2}} \frac{h v}{k_{\mathrm{B}} T_{0}} \int_{0}^{R} \nabla \cdot \boldsymbol{\xi} r_{0} \mathrm{~d} r_{0}+2 \frac{\xi_{r}(R)}{R}$.

Substituting $\nabla \cdot \xi$ in terms of $P^{\prime}$ shows

$\frac{\delta \mathcal{I}}{\mathcal{I}_{0}}=2\left\{\frac{h v}{k_{\mathrm{B}} T_{0}} \frac{(\gamma-1) \omega^{2}}{\rho_{0}\left(c_{\mathrm{s}}^{2}+c_{\mathrm{A}}^{2}\right)\left(\omega^{2}-k^{2} c_{\mathrm{T}}^{2}\right)} \frac{1}{R^{2}} \int_{0}^{R} P^{\prime} r_{0} \mathrm{~d} r_{0}+\frac{\xi_{r}(R)}{R}\right\}$

In the above equation $c_{\mathrm{T}}$ is the tube speed, defined as

$c_{\mathrm{T}}=\frac{c_{\mathrm{s}} c_{\mathrm{A}}}{\left(c_{\mathrm{s}}^{2}+c_{\mathrm{A}}^{2}\right)^{1 / 2}}$

where $c_{\mathrm{S}}=\left(\gamma p_{0} / \rho_{0}\right)^{1 / 2}$ and $c_{\mathrm{A}}=B_{0} /\left(\mu \rho_{0}\right)^{1 / 2}$ are the sound and Alfvén speeds. Equation (3) becomes

$\frac{\delta \phi_{B}}{\phi_{B, 0}}=2\left\{\frac{\left(\omega^{2}-k^{2} c_{\mathrm{s}}^{2}\right)}{\rho_{0}\left(c_{\mathrm{s}}^{2}+c_{\mathrm{A}}^{2}\right)\left(\omega^{2}-k^{2} c_{\mathrm{T}}^{2}\right)} \frac{1}{R^{2}} \int_{0}^{R} P^{\prime} r_{0} \mathrm{~d} r_{0}+\frac{\xi_{r}(R)}{R}\right\}$.

We are interested in the phase behaviour of the integrated intensity and the cross-sectional area variations and therefore focus on Eqs. (1) and (7). We start with a surface mode with $P^{\prime}=I_{0}(\kappa r)$ where $\kappa$ is given by

$\kappa^{2}=\frac{\left(k^{2} c_{\mathrm{s}}^{2}-\omega^{2}\right)\left(k^{2} c_{\mathrm{A}}^{2}-\omega^{2}\right)}{\left(c_{\mathrm{s}}^{2}+c_{\mathrm{A}}^{2}\right)\left(k^{2} c_{\mathrm{T}}^{2}-\omega^{2}\right)}$.

Note that $\kappa^{2}$ is positive for surface modes. The radial component of the Lagrangian displacement

$\xi_{r}=\frac{1}{\rho_{0}\left(\omega^{2}-k^{2} c_{\mathrm{A}}^{2}\right)} \frac{\mathrm{d} P^{\prime}}{\mathrm{d} r}=\frac{\kappa}{\rho_{0}\left(\omega^{2}-k^{2} c_{\mathrm{A}}^{2}\right)} I_{1}(\kappa r)$.

We can easily calculate $\int_{0}^{R} P^{\prime} r_{0} \mathrm{~d} r_{0}$ using identity 11.3 .25 from Abramowitz \& Stegun (1972) and find

$$
\int_{0}^{R} P^{\prime} r_{0} \mathrm{~d} r_{0}=\frac{R}{\kappa} I_{1}(\kappa R) .
$$

Thus Eqs. (1), (7), and (8) become

$$
\begin{aligned}
& \frac{\delta \mathcal{I}}{\mathcal{I}_{0}}=2\left\{\frac{h v}{k_{\mathrm{B}} T_{0}} \frac{(\gamma-1) \omega^{2}}{\rho_{0}\left(c_{\mathrm{s}}^{2}+c_{\mathrm{A}}^{2}\right)\left(\omega^{2}-k^{2} c_{\mathrm{T}}^{2}\right)}+\frac{\kappa^{2}}{\rho_{0}\left(\omega^{2}-k^{2} c_{\mathrm{A}}^{2}\right)}\right\} \frac{I_{1}(\kappa R)}{\kappa R} \\
& \frac{\delta S}{S_{0}}=\frac{2 \kappa^{2}}{\rho_{0}\left(\omega^{2}-k^{2} c_{\mathrm{A}}^{2}\right)} \frac{I_{1}(\kappa R)}{\kappa R} \\
& \frac{\delta \phi_{B}}{\phi_{B, 0}}=2\left\{\frac{\left(\omega^{2}-k^{2} c_{\mathrm{s}}^{2}\right)}{\rho_{0}\left(c_{\mathrm{s}}^{2}+c_{\mathrm{A}}^{2}\right)\left(\omega^{2}-k^{2} c_{\mathrm{T}}^{2}\right)}+\frac{\kappa^{2}}{\rho_{0}\left(\omega^{2}-k^{2} c_{\mathrm{A}}^{2}\right)}\right\} \frac{I_{1}(\kappa R)}{\kappa R}
\end{aligned}
$$

This last equation can be simplified by substituting $\kappa^{2}$ and shows $\frac{\delta \phi_{B}}{\phi_{B, 0}}=0$. Why the Lagrangian variation of the magnetic flux is zero is obvious. We have calculated the Lagrangian variation of the magnetic flux over a surface $S$ that moves with the plasma. In ideal MHD the magnetic field lines are frozen in the plasma, thus the variation of the magnetic flux is zero. This result clearly shows that the observations in Fujimura \& Tsuneta (2009), which have a non-zero magnetic flux perturbation, were taken in an Eulerian way and not in a Lagrangian way. The authors claimed that they tracked the region of interest (ROI), e.g. a magnetic pore, in a Lagrangian way for an extended period of time. However, they also explained that for a magnetic pore the ROI was typically only a portion of the pore and the size of the ROI did not change in time. This last statement clearly shows that when the magnetic pore expanded, the ROI did not. This confirms that it was justified to study Eulerian perturbations in Moreels \& Van Doorsselaere (2013), since the goal was to apply the model to the observations made in Fujimura \& Tsuneta (2009).

We now turn to body modes with $P^{\prime}=J_{0}(n r)$ and

$\xi_{r}=\frac{-n}{\rho_{0}\left(\omega^{2}-k^{2} c_{\mathrm{A}}^{2}\right)} J_{1}(n r)$,

where $n^{2}=-\kappa^{2}$ and is again positive. Using identity 11.3.20 from Abramowitz \& Stegun (1972), we find

$$
\int_{0}^{R} P^{\prime} r_{0} \mathrm{~d} r_{0}=\frac{R}{n} J_{1}(n R) .
$$

Thus Eqs. (1), (7), and (8) become

$$
\begin{aligned}
& \frac{\delta \mathcal{I}}{\mathcal{I}_{0}}=2\left\{\frac{h v}{k_{\mathrm{B}} T_{0}} \frac{(\gamma-1) \omega^{2}}{\rho_{0}\left(c_{\mathrm{s}}^{2}+c_{\mathrm{A}}^{2}\right)\left(\omega^{2}-k^{2} c_{\mathrm{T}}^{2}\right)}+\frac{\kappa^{2}}{\rho_{0}\left(\omega^{2}-k^{2} c_{\mathrm{A}}^{2}\right)}\right\} \frac{J_{1}(n R)}{n R}(12) \\
& \frac{\delta S}{S_{0}}=\frac{2 \kappa^{2}}{\rho_{0}\left(\omega^{2}-k^{2} c_{\mathrm{A}}^{2}\right)} \frac{J_{1}(n R)}{n R} \\
& \frac{\delta \phi_{B}}{\phi_{B, 0}}=2\left\{\frac{\left(\omega^{2}-k^{2} c_{\mathrm{s}}^{2}\right)}{\rho_{0}\left(c_{\mathrm{s}}^{2}+c_{\mathrm{A}}^{2}\right)\left(\omega^{2}+k^{2} c_{\mathrm{T}}^{2}\right)}+\frac{\kappa^{2}}{\rho_{0}\left(\omega^{2}-k^{2} c_{\mathrm{A}}^{2}\right)}\right\} \frac{J_{1}(n R)}{n R} \cdot(14)
\end{aligned}
$$

This last equation can be simplified by substituting $\kappa^{2}$ and as expected $\frac{\delta \phi_{B}}{\phi_{B, 0}}=0$. Note that the terms in Eqs. (12)-(14) before $J_{1}(n R) / n R$ are the same as the terms in Eqs. (9)-(11) before $I_{1}(\kappa R) / \kappa R$. We shall use $I_{1}$ to indicate the first term in Eq. (9), $\mathcal{I}_{2}$ to indicate the second term in Eq. (9), and $S_{1}$ to indicate the first term in Eq. (10). Looking at Eqs. (9) and (10), it is clear that for surface modes the intensity and the cross-sectional area variations are either in phase or in antiphase, depending only on the sign in front of $I_{1}(\kappa R) / \kappa R$ in Eqs. 9 and 10. A similar argument holds for body modes.

The signs of $\mathcal{I}_{1}, \mathcal{I}_{2}$, and $S_{1}$ can be determined by looking at a phase diagram for wave modes under photospheric conditions (Edwin \& Roberts 1983). The result is shown in Fig. 2. To obtain this phase diagram we used an equilibrium model with $c_{\mathrm{A}, \mathrm{i}}=2 c_{\mathrm{s}, \mathrm{i}}, c_{\mathrm{A}, \mathrm{e}}=0.5 c_{\mathrm{s}, \mathrm{i}}$, and $c_{\mathrm{s}, \mathrm{e}}=1.5 c_{\mathrm{s}, \mathrm{i}}$. We can determine different wave modes. Starting from the top, we first have the fast surface mode, second we have slow body modes, and third we have the slow surface mode. For the slow body modes we plotted two lines corresponding to different radial overtones. For more information on these modes and their eigenfunctions see Moreels \& Van Doorsselaere (2013). 
M. G. Moreels et al.: Cross-sectional area and intensity variations of sausage modes

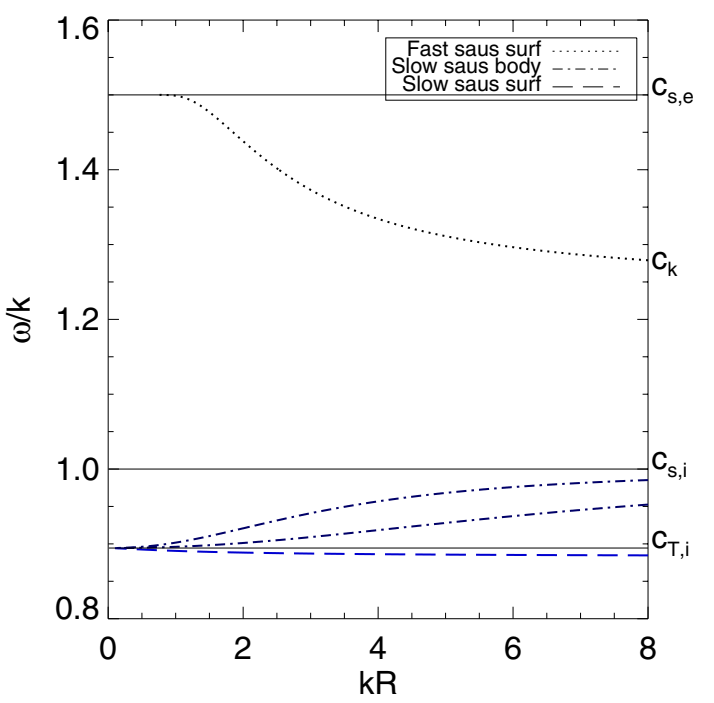

Fig. 2. Phase speed diagram of wave modes under photospheric conditions. We have taken $c_{\mathrm{A}, \mathrm{i}}=2 c_{\mathrm{s}, \mathrm{i}}, c_{\mathrm{A}, \mathrm{e}}=0.5 c_{\mathrm{s}, \mathrm{i}}$, and $c_{\mathrm{s}, \mathrm{e}}=1.5 c_{\mathrm{s}, \mathrm{i}}$. The Alfvén speeds are not indicated in the graph because no modes with real frequencies appear in that vicinity. The modes with phase speeds between $c_{\mathrm{T}, \mathrm{i}}$ and $c_{\mathrm{s}, \mathrm{i}}$ are body modes and the other modes are surface modes. Note that we only plotted two body modes, while there are infinitely many radial overtones.

Looking at the phase diagram (Fig. 2), we find that for the slow surface mode $\mathcal{I}_{1}, \mathcal{I}_{2}$, and $S_{1}$ are all negative. This shows that the intensity and the cross-sectional area variations are in phase for a slow surface mode. For a slow body mode (i.e. $\kappa^{2}<0$ ) we find that $\mathcal{I}_{1}, \mathcal{I}_{2}$, and $S_{2}$ are all positive, showing that the intensity and the cross-sectional area variations are in phase for a slow body mode. For fast surface modes we find that $\mathcal{I}_{1}$ is positive while $\mathcal{I}_{2}$ and $S_{2}$ are negative. Thus we do not yet know if the intensity and the cross-sectional area variations are in phase or in antiphase. Therefore we look at the ratio of the $I_{1}$ to $I_{2}$ and find

$\left|\frac{I_{1}}{I_{2}}\right|=\frac{h v}{k_{\mathrm{B}} T_{0}} \frac{(\gamma-1) \omega^{2} / k^{2}}{\left|c_{\mathrm{s}}^{2}-\omega^{2} / k^{2}\right|}$.

Because we have integrated the intensity over the interior of the flux tube, we substitute $T_{0}$ with $T_{\mathrm{i}}$, which is the equilibrium temperature inside the flux tube. Because the phase speed $\omega / k$ decreases when $k R$ increases (see Fig. 2), we know that $\left|\mathcal{I}_{1} / I_{2}\right|$ increases when $k R$ increases. We therefore look at the thin tube limit (i.e. $k R \ll 1$ ), for which we know $\omega / k \rightarrow c_{\mathrm{s}, \mathrm{e}}$, and find

$\left|\frac{\mathcal{I}_{1}}{\mathcal{I}_{2}}\right|=\frac{h v}{k_{\mathrm{B}} T_{\mathrm{i}}} \frac{(\gamma-1)}{\left|T_{\mathrm{i}} / T_{\mathrm{e}}-1\right|}$,

where $T_{\mathrm{i}}$ and $T_{\mathrm{e}}$ are the internal and external equilibrium temperatures. In this derivation we used that the sound speeds are proportional to the temperature. Typical values for the photosphere can be found in the literature (Fujimura \& Tsuneta 2009; Morton et al. 2011), and using these, we find

$\left|\frac{I_{1}}{I_{2}}\right|>1$

showing that $\mathcal{I}_{1}$ is higher in absolute value than $\mathcal{I}_{2}$. The sign of $\mathcal{I}_{1}$ is positive while the sign of $S_{1}$ is negative and thus we have an antiphase behaviour.

We now investigate the equilibrium temperatures we need to have $\left|\mathcal{I}_{1} / I_{2}\right|<1$ in Eq. (15). Because the internal temperature is
Table 1. Phase differences between the cross-sectional area variation and the intensity perturbation for different sausage wave modes.

\begin{tabular}{l|ccc|l}
\hline \hline Wave mode & sign of $\mathcal{I}_{1}$ & Sign of $\mathcal{I}_{2}$ & sign of $S_{1}$ & Phase behaviour \\
\hline Slow surface & - & - & - & in phase \\
Slow body & + & + & + & in phase \\
Fast surface & + & - & - & in antiphase \\
\hline
\end{tabular}

lower that the external temperature for photospheric flux tubes, we can rewrite $\left|\mathcal{I}_{1} / \mathcal{I}_{2}\right|<1$ to

$0<-(\gamma-1) \frac{h c}{k_{\mathrm{B}} \lambda}+T_{\mathrm{i}}-\frac{1}{T_{\mathrm{e}}} T_{\mathrm{i}}^{2}=f\left(T_{\mathrm{i}}\right)$,

where $\lambda$ is the wavelength of the observations. For this inequality to be valid, $f\left(T_{\mathrm{i}}\right)$ must have a real zero, i.e. the discriminant must be positive, resulting in

$\lambda T_{\mathrm{e}}>4(\gamma-1) \frac{h c}{k_{\mathrm{B}}}=0.038$.

Typical values for the wavelength $\lambda$ in the photosphere are 400 to $650 \mathrm{~nm}$ (Fujimura \& Tsuneta 2009; Morton et al. 2011), indicating exteral temperatures of the order $7 \times 10^{4} \mathrm{~K}$, which are unrealistic for the photosphere. This argument shows that for the photosphere $\left|I_{1} / I_{2}\right|>1$, and thus we have an antiphase behaviour between the cross-sectional area and the intensity variation for fast sausage modes. These results are summarised in Table 1.

\section{Visualisations}

We show visualisations for different sausage modes to illustrate the phase differences. We computed sausage modes using the same equilibrium values as before, namely $c_{\mathrm{A}, \mathrm{i}}=2 c_{\mathrm{S}, \mathrm{i}}$, $c_{\mathrm{A}, \mathrm{e}}=0.5 c_{\mathrm{s}, \mathrm{i}}$, and $c_{\mathrm{s}, \mathrm{e}}=1.5 c_{\mathrm{s}, \mathrm{i}}$. We fixed the value of $k R=2$ and fixed the external density $\rho_{0, \mathrm{e}}=1$. The first mode is the fast surface mode; the result can be seen in Fig. 3. There are two panels in this figure, representing different times in the computation. The left panel is at $t=0$ and the right panel is at $t=P / 2$, where $P$ is the period of the wave. The background colour indicates the density, which is a proxy for the intensity, the flux-tube boundary is indicated by a black line, and the vectors indicate the Lagrangian displacement. We notice that when the surface area decreases, i.e. the radius of the flux tube decreases, the intensity increases. This is no confirmation of the antiphase behaviour, because the intensity increases but the area over which the intensity is integrated decreases. Therefore we have plotted the radial displacement of the flux-tube boundary and the total intensity over the flux tube (see Fig. 4). This figure clearly demonstrates the antiphase behaviour.

The second mode is the slow body mode; the result can be seen in Fig. 5. This figure contains two panels corresponding to different times in the computation. The left panel is at $t=0$ and the right panel is at $t=P / 2$. As before, the background colour indicates the density, which is a proxy for the intensity, the flux-tube boundary is indicated by a black line, and the vectors indicate the displacement. We notice that when the surface area decreases, i.e. the radius of the flux decreases, the intensity also decreases. Thus, we can conclude that the total intensity decreases when the surface area decreases, confirming the in-phase behaviour of slow body modes.

The third mode is the slow surface mode; the result can be seen in Fig. 6. In this figure there are two panels corresponding 


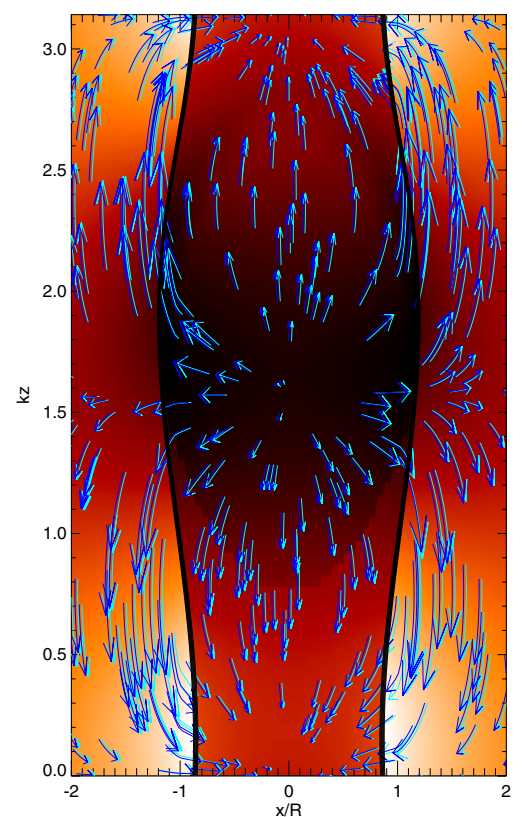

(a) Fast sausage surface wave at $t=0$.

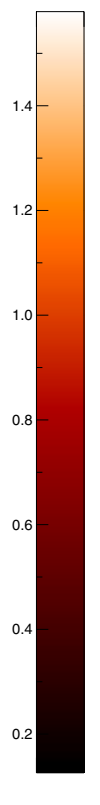

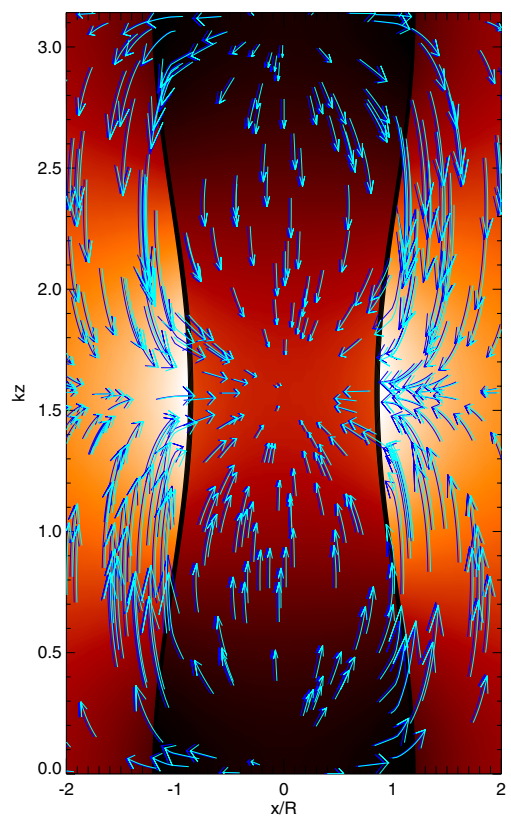

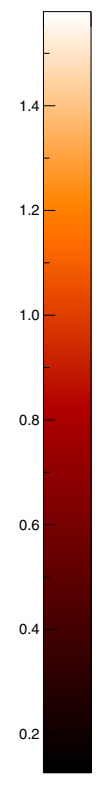

(b) Fast sausage surface wave at $t=P / 2$.

Fig. 3. Longitudinal cut of a fast sausage surface wave at two different times. The background colour indicates the density, which is used as a proxy for the intensity. The flux-tube boundary is indicated by a black line. The arrows indicate the plasma displacement.

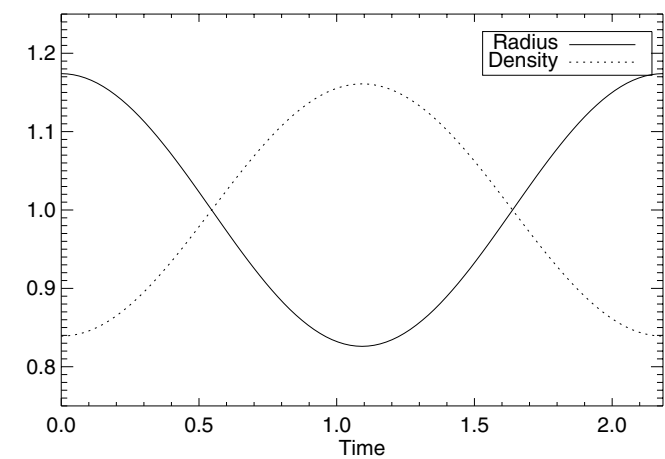

Fig. 4. The radial displacement of the flux-tube boundary and the total intensity over the flux tube as a function of time at a fixed height for the fast surface mode. The displacement and the intensity vary in antiphase with one another.

to different times in the computation. The left panel is at $t=0$ and the right panel is at $t=P / 2$. The background colour indicates the density, which is a proxy for the density, the flux-tube boundary is indicated by a black line, and the vectors indicate the displacement. We notice that when the surface area decreases, i.e. the radius of the flux decreases, the intensity also decreases. Thus, we can conclude that the total intensity decreases when the surface area decreases, confirming the in-phase behaviour of slow surface modes.

We emphasise that Figs. 5 and 6 show that for slow modes the longitudinal displacement is much more pronounced than the radial displacement. This indicates that it is unlikely that the surface area variation is observable for slow modes. This result is also confirmed in Moreels \& Van Doorsselaere (2013), where we showed that the polarisation for slow modes in highly longitudinal. Figure 5 (Moreels \& Van Doorsselaere 2013) clearly shows that the longitudinal displacement $\xi_{\|}$is much higher than the transverse displacement $\xi_{\perp}$ for slow modes. Another confirmation are the data in Fujimura \& Tsuneta (2009). From the observations in Fujimura \& Tsuneta (2009) we find that a typical value for the radius of the flux tube $R$ is $2000 \mathrm{~km}$. We also find that the line-of-sight velocity amplitude is of the order $80 \mathrm{~m} / \mathrm{s}$ and the period is of the order $350 \mathrm{~s}$. Since $\boldsymbol{v}=\partial \boldsymbol{\xi} / \partial t=\mathrm{i} \omega \boldsymbol{\xi}$, the amplitude of $\xi_{r}$ is equal to the amplitude of the velocity perturbation divided by the angular frequency. Using the values in Fujimura \& Tsuneta (2009), we find that the order of magnitude of the amplitude of $\xi_{r}$ is $4.5 \mathrm{~km}$, which is below the current resolution of the observations. Based on these three sources we conclude that if we observe a surface area variation, we are most likely observing a fast sausage mode. The fact that the longitudinal displacement is much higher than the radial displacement for slow modes also justifies the use of the simple one-dimensional approach for modelling slow magnetoaccoustic oscillations in the chromosphere, as in Botha et al. (2011).

Finally, we briefly discuss the observations taken in Morton et al. (2011). In that article observations for magnetic pores were reported and the authors compared the variations in pore size with the variations in intensity. They concluded that the observations were sausage modes but were not able to determine the wave mode, i.e. slow or fast. The authors are convinced that the observations had a strong out-of-phase behaviour. Applying our model to these observations shows that the observations are fast sausage modes. This is also confirmed by the previous paragraph where we showed that if we observe a cross-sectional area variation, we are most likely dealing with a fast mode.

\section{Conclusions}

We have shown that cross-sectional area variations and intensity variations are in phase for slow sausage modes while they are in antiphase for fast sausage modes. We did this by modelling the flux tube as a straight cylinder with constant radius and constant plasma parameters both inside and outside the flux tube. We then calculated the cross-sectional area and the integrated intensity variations. From these variations we were able to deduce 
M. G. Moreels et al.: Cross-sectional area and intensity variations of sausage modes

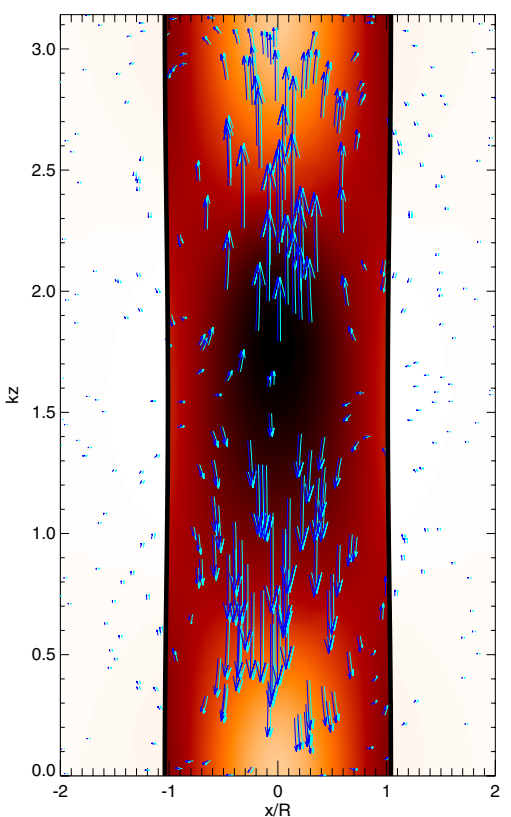

(a) Slow sausage body wave at $t=0$.
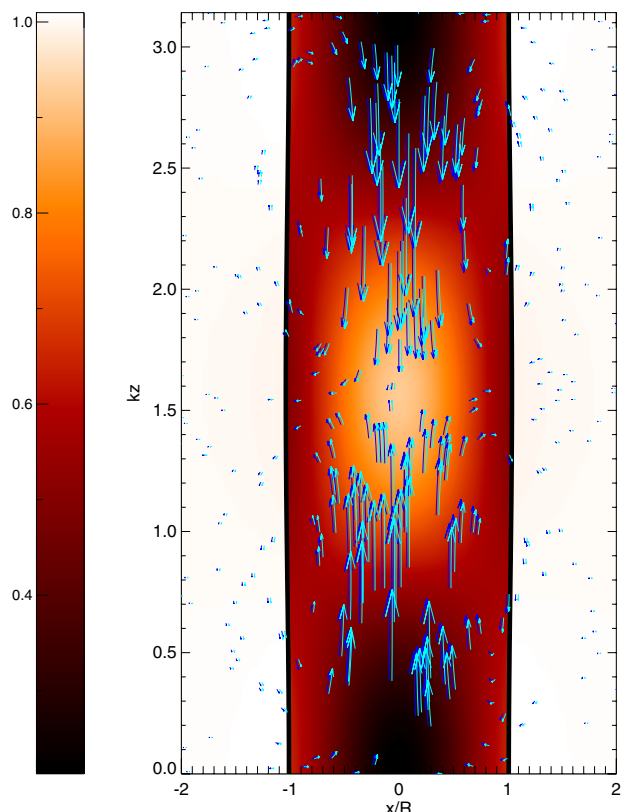

(b) Slow sausage body wave at $t=P / 2$.

Fig. 5. Longitudinal cut of a slow sausage body wave at two different times. The background colour indicates the density, which is used as a proxy for the intensity. The flux-tube boundary is indicated by a black line. The arrows indicate the plasma displacement.

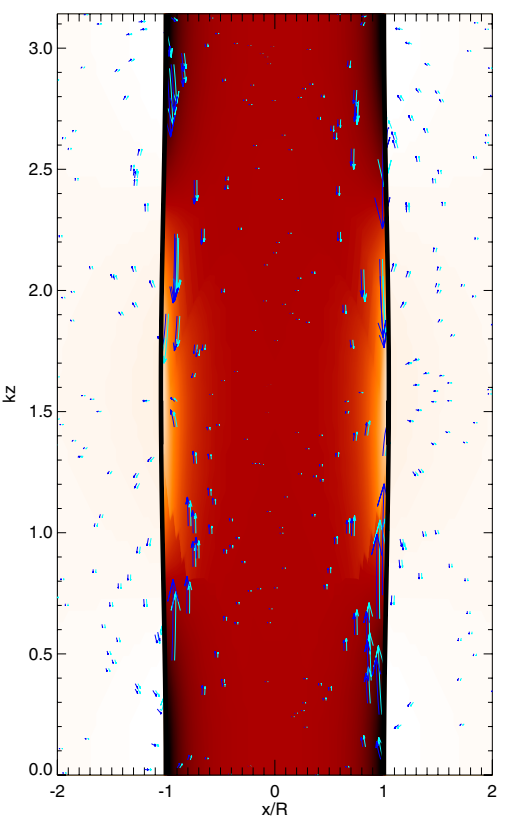

(a) Slow sausage surface wave at $t=0$.
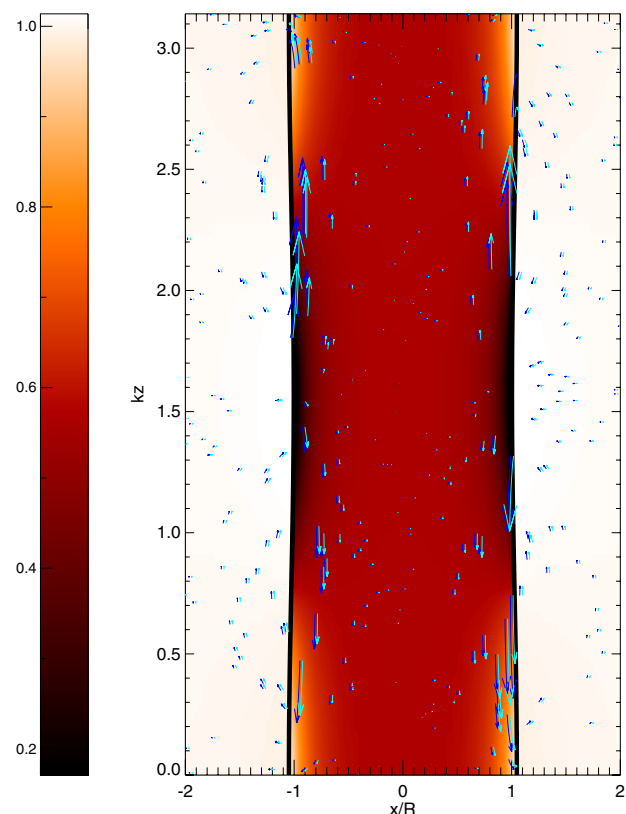

(b) Slow sausage surface wave at $t=P / 2$.

Fig. 6. Longitudinal cut of a slow sausage surface wave at two different times. The background colour indicates the density, which is used as a proxy for the intensity. The flux-tube boundary is indicated by a black line. The arrows indicate the plasma displacement.

the phase difference between the variations. We verified these results using some visualisations.

In our derivation we found that the Lagrangian perturbation of the magnetic flux is zero. This was to be expected in ideal MHD. The surface over which we integrated the magnetic flux was following the motion of the plasma, and in ideal MHD the magnetic field lines are frozen in the plasma. This result showed that the ROI used in Fujimura \& Tsuneta (2009) to study the photospheric pore oscillations did not follow the plasma motions perfectly. Their observations are probably better described with Eulerian variations (e.g. Moreels \& Van Doorsselaere 2013).

The visualisations showed that for slow modes the longitudinal displacement is much higher than the transverse displacement. This result was also found in Moreels \& Van Doorsselaere (2013) by studying the polarisation of different wave modes. Another confirmation of this result was obtained from the observations in Fujimura \& Tsuneta (2009), from which we deduced that the order of magnitude of the amplitude of $\xi_{r}$ is 
$4.5 \mathrm{~km}$, which is below the current resolution of the observations. Therefore we conclude that if one observes a crosssectional area variation, the most likely interpretation is a fast sausage mode.

Finally, we also applied our model to the observations in Morton et al. (2011), where the authors claimed to find an out-ofphase behaviour between variations in the cross-sectional area and the intensity. The fact that the cross-sectional area variation is observable indicates that the observations are most likely fast sausage modes. The out-of-phase behaviour confirms that the observation is a fast sausage mode.

Acknowledgements. We have received funding from the Odysseus programme of the FWO-Vlaanderen and from the EU's Framework Programme 7 as an ERG with grant No. 276808. This research is made in the context of the Interuniversity Attraction Poles Programme initiated by the Belgian Science Policy Office (IAP P7/08 CHARM). Marcel Goossens acknowledges GOA/2009-09.

\section{References}

Abramowitz, M., \& Stegun, I. A. 1972, Handbook of Mathematical Functions, eds. M. Abramowitz, \& I. A. Stegun (New York: Dover)

Andries, J., \& Cally, P. S. 2011, ApJ, 743, 164

Banerjee, D., Pérez-Suárez, D., \& Doyle, J. G. 2009, A\&A, 501, L15

Botha, G. J. J., Arber, T. D., Nakariakov, V. M., \& Zhugzhda, Y. D. 2011, ApJ, 728,84

Dorotovič, I., Erdélyi, R., \& Karlovský, V. 2008, in IAU Symp. 247, eds. R. Erdélyi, \& C. A. Mendoza-Briceno, 351
Edwin, P. M., \& Roberts, B. 1983, Sol. Phys., 88, 179

Erdélyi, R., Malins, C., Tóth, G., \& de Pontieu, B. 2007, A\&A, 467, 1299

Fujimura, D., \& Tsuneta, S. 2009, ApJ, 702, 1443

Jess, D. B., Pascoe, D. J., Christian, D. J., et al. 2012a, ApJ, 744, L5

Jess, D. B., Shelyag, S., Mathioudakis, M., et al. 2012b, ApJ, 746, 183

Leighton, R. B. 1960, in Aerodynamic Phenomena in Stellar Atmospheres, ed. R. N. Thomas, IAU Symp., 12, 321

Liu, W., Nitta, N. V., Schrijver, C. J., Title, A. M., \& Tarbell, T. D. 2010, ApJ, 723, L53

Marshall, K. P. 2003, Astron. Geophys., 44, 050000

Moreels, M. G., \& Van Doorsselaere, T. 2013, A\&A, 551, A137

Morton, R. J., Erdélyi, R., Jess, D. B., \& Mathioudakis, M. 2011, ApJ, 729, L18

Morton, R. J., Verth, G., McLaughlin, J. A., \& Erdélyi, R. 2012, ApJ, 744, 5

Nakariakov, V. M., Ofman, L., Deluca, E. E., Roberts, B., \& Davila, J. M. 1999, Science, 285,862

Osterbrock, D. E. 1961, ApJ, 134, 347

Pradhan, A. K., \& Nahar, S. N. 2011, Atomic Astrophysics and Spectroscopy (Cambridge: Cambridge University Press)

Roberts, B., \& Webb, A. R. 1978, Sol. Phys., 56, 5

Rosenthal, C. S., Bogdan, T. J., Carlsson, M., et al. 2002, ApJ, 564, 508

Rutten, R. J. 2003, Radiative Transfer in Stellar Atmospheres (New York: John Wiley)

Rybicki, G. B., \& Lightman, A. P. 1986, Radiative Processes in Astrophysics (Wiley)

Sakurai, T., Goossens, M., \& Hollweg, J. V. 1991, Sol. Phys., 133, 227

Stein, R. F., \& Leibacher, J. 1974, ARA\&A, 12, 407

Thompson, B. J., Gurman, J. B., Neupert, W. M., et al. 1999, ApJ, 517, L151

Tomczyk, S., McIntosh, S. W., Keil, S. L., et al. 2007, Science, 317, 1192

Van Doorsselaere, T., Nakariakov, V. M., \& Verwichte, E. 2008, ApJ, 676, L73 


\section{Appendix A: Surface with constant optical depth}

The optical depth $\tau_{v}$ can be calculated using (Rybicki \& Lightman 1986; Rutten 2003)

$\tau_{v}=\int_{0}^{D} \alpha_{v} \mathrm{~d} s$

This shows that the optical depth depends on the frequency $v$, the LOS, and the extinction coefficient $\alpha$, which also depends on the photon frequency. To simplify the calculations we assume that the LOS is directed along the $z$-axis, i.e. $\mathrm{d} s=\mathrm{d} z$. The goal is to find the height $D$ for which the optical depth at a given frequency $\tau_{v}$ is constant. Because we are using observations of the photosphere, we assume that the intensity observations are taken in the continuum and not in the line core. In Rutten (2003) we can find different sources for opacity in the continuum each with their own extinction coefficient. We have bound-free transitions, free-free transitions, Thomson scattering, and Rayleigh scattering. The most important source for the solar photosphere comes from bound-free transitions where the hydride $H^{-}$absorb radiation to form hydrogen and a free electron (Marshall 2003; Pradhan \& Nahar 2011). From Rutten (2003) we find that for bound-free transitions $\alpha_{v}=\sigma n_{i}\left\{1-\exp \left(-\frac{h v}{k_{\mathrm{B}} T}\right)\right\}$, where $\sigma$ is a constant that depends on the atom/ion involved in the bound-free transition, $n_{i}$ is the number density in the ionising level, $h$ is the Planck constant, $k_{\mathrm{B}}$ is the Boltzmann constant, $T$ is the temperature of the plasma, and $v$ is the frequency. We assume that $n_{i}$ is proportional to the density $\rho$, thus the monochromatic optical depth is

$\tau_{v}=a \sigma \int_{0}^{D} \rho\left\{1-\exp \left(-\frac{h v}{k_{\mathrm{B}} T}\right)\right\} \mathrm{d} z$,

where $a$ indicates the fraction of ionisation. We can now linearise both the density and the temperature to find

$\rho\left\{1-\exp \left(-\frac{h v}{k_{\mathrm{B}} T}\right)\right\} \approx \rho_{0}\left(1-\mathcal{I}_{0}\right)+\rho_{0} A(r) P^{\prime}+\rho_{0} B(r) \xi_{r}$,

where $\mathcal{I}_{0}, A(r)$, and $B(r)$ are given by

$$
\begin{aligned}
& \mathcal{I}_{0}(r)=2 \frac{h v^{3}}{c^{2}} \exp \left(-\frac{h v}{k_{\mathrm{B}} T_{0}(r)}\right) \\
& A(r)=\frac{\omega^{2}}{\rho_{0}\left(c_{\mathrm{S}}^{2}+c_{\mathrm{A}}^{2}\right)\left(\omega^{2}-k^{2} c_{\mathrm{T}}^{2}\right)}\left(\left(1-\mathcal{I}_{0}\right)-(\gamma-1) \frac{h v}{k_{\mathrm{B}} T_{0}(r)} \mathcal{I}_{0}\right) \\
& B(r)=-\frac{h v}{k_{\mathrm{B}} T_{0}(r)} \mathcal{I}_{0}\left(\frac{1}{\rho_{0}} \frac{\mathrm{d} \rho_{0}}{\mathrm{~d} r}-\frac{1}{p_{0}} \frac{\mathrm{d} p_{0}}{\mathrm{~d} r}\right)-\left(1-\mathcal{I}_{0}\right) \frac{1}{\rho_{0}} \frac{\mathrm{d} \rho_{0}}{\mathrm{~d} r} .
\end{aligned}
$$

The optical depth (Eq. (A.1)) is thus given by

$\tau_{v}=a \sigma \rho_{0}\left[\left(1-\mathcal{I}_{0}\right) \int_{0}^{D} \mathrm{~d} z+A(r) \int_{0}^{D} P^{\prime} \mathrm{d} z+B(r) \int_{0}^{D} \xi_{r} \mathrm{~d} z\right]$.

In general, $D$ is a function of $r, \varphi$, and $t$, but since we are dealing with axisymmetric modes, we have $D(r, t)$. We linearise $D(r, t)=$ $D_{0}+D^{\prime}(r, t)$ and substitute in Eq. (A.2) to find

$$
\tau_{v}=a \sigma \rho_{0}\left[\left(1-\mathcal{I}_{0}\right)\left(D_{0}+D^{\prime}\right)+A(r) \int_{0}^{D_{0}} P^{\prime} \mathrm{d} z+B(r) \int_{0}^{D_{0}} \xi_{r} \mathrm{~d} z\right],
$$

where we neglected higher-order terms. Because the optical depth $\tau_{v}$ is constant, the zeroth-order terms in Eq. (A.3) show

$D_{0}(r)=\frac{\tau_{v}}{a \sigma \rho_{0}\left(1-\mathcal{I}_{0}\right)}$

To find $D^{\prime}$ we look at the first-order terms in Eq. (A.3) and find

$D^{\prime}(r, t)=\frac{-1}{\left(1-\mathcal{I}_{0}\right)}\left(A(r) \int_{0}^{D_{0}} P^{\prime} \mathrm{d} z+B(r) \int_{0}^{D_{0}} \xi_{r} \mathrm{~d} z\right)$.

Since both $P^{\prime}$ and $\xi_{r}$ are proportional to $\exp \{\mathrm{i}(k z-\omega t)\}$,

$D^{\prime}(r, t)=\frac{-1}{\left(1-I_{0}\right)}\left(A(r) P^{\prime}(r, t)+B(r) \xi_{r}(r, t)\right) \frac{\exp \left(\mathrm{i} k D_{0}\right)-1}{\mathrm{i} k}$.

The equation for a surface with constant optical depth is given by $g(r, z, t) \equiv z-D(r, t)$. In the uniform case we have $D_{0}$ constant, $A(r)$ constant, and $B(r)=0$, thus $g(r, z, t)$ has a similar form as the surface that follows the motions of the plasma (i.e. $\left.f(r, \varphi, z, t) \equiv z-\xi_{z}(r, \varphi, z=0, t)\right)$. We calculate the normal to the surface $g$

$\mathbf{1}_{n} \sim\left(\frac{A}{\left(1-I_{0}\right)} \frac{\left(\exp \left(\mathrm{i} k D_{0}\right)-1\right)}{\mathrm{i} k} \frac{\partial P^{\prime}}{\partial r} \mathbf{1}_{\boldsymbol{r}}+\mathbf{1}_{z}\right)$.

We relate a surface element $\mathrm{d} S$ to an elemental surface element in the horizontal plane $\mathrm{d} S_{\mathrm{h}}$, i.e.

$\mathrm{d} S=\mathrm{d} S_{\mathrm{h}} \sqrt{1+\left(\frac{A}{\left(1-\mathcal{I}_{0}\right)} \frac{\left(\exp \left(\mathrm{i} k D_{0}\right)-1\right)}{\mathrm{i} k} \frac{\partial P^{\prime}}{\partial r}\right)^{2}}$,

which in a linear approximation is $\mathrm{d} S=\mathrm{d} S_{\mathrm{h}}=r \mathrm{~d} r \mathrm{~d} \varphi$. This shows that integrating over the surface where $\tau_{v}$ is constant or integrating over the surface that follows the motions of the plasma is identical, since for a uniform equilibrium and in a linear expansion the elemental surface elements are the same. 